\title{
Sind Tatsachen vom Sprachspiel konstituiert? \\ Zu Wittgensteins Philosophie 1946-51
}

Katalin Neumer, Budapest

\section{Einleitung}

Die Spätkonzeption Wittgensteins zählt man öfters, und nicht nur außerhalb, sondern auch innerhalb der Wittgenstein-Forschung, zu jenen, die für eine überwältigende Macht unserer begrifflichen Schemata bzw. unserer Kultur und Lebensform plädierten, und zwar sogar in dem Sinne, dass sie im Hinblick auf die Tatsachen dermaßen bestimmend wären, dass die letzteren von ihnen konstituiert sind (was auch immer der Ausdruck ,konstituieren" bedeuten könnte). Dieser Interpretationstrend lässt sich wohl u.a. auf ideengeschichtliche Gründe zurückführen, aber auch mit etlichen Textstellen Wittgensteins untermauern wie auch mit der folgenden:

Wir sind an eine bestimmte Einteilung der Sachen gewöhnt.

Sie ist uns mit der Sprache, oder den Sprachen, zur Natur geworden.

Dies sind die festen Schienen, auf denen all unser Denken verläuft, und also nach ihnen auch unser Urteilen und Handeln. (MS 137: 64b-65a; vgl. TS 232: 763-764, TS 233b: 4)

Oder auch mit Passagen aus den Philosophischen Untersuchungen wie z.B.:

Welche Art von Gegenstand etwas ist, sagt die Grammatik. (PU, § 373)

Die Frage ist freilich, ob diese „Einteilung der Sachen“, die „festen Schienen“ der Sprache, die „Grammatik“, unsere Sprachspiele mehr oder weniger von der Wirklichkeit bedingt oder aber eben umgekehrt, vollkommen willkürlich in Bezug auf die Wirklichkeit sind. Letzteres gilt als (wenn auch alleine noch nicht ausreichende) Bedingung dafür, eine eindeutig bejahende Antwort auf unsere Ausgangsfrage geben zu können. 
Im folgenden werde ich mich auf Bemerkungen konzentrieren, die Wittgenstein nach den Philosophischen Untersuchungen zu Papier gebracht hat: In dieser Periode kommt er auffallend häufig auf Fragen zu sprechen, inwieweit Fakten von Begriffen bzw. von den Sprachspielen bestimmt sind, und auch umgekehrt: ob sie einen Einfluss auf unsere Begriffsbildung ausüben könnten. Dabei erhebt sich die Frage, ob es nur um ein verstärktes Interesse für seine alten Fragen geht, oder aber auch eine Veränderung oder wenigstens Modifizierung seiner früheren Konzeption stattfindet. Somit berührt mein Aufsatz auch das heutzutage breit diskutierte Thema eines „Third Wittgenstein“. Der an dieser Stelle erlaubte Umfang lässt mich nur skizzenhaft vorgehen: Ich werde vorerst kurz einige Ausführungen Wittgensteins aus seinen letzten Jahren darstellen, die nahelegen, dass Tatsachen auch Eigenwert haben können. Obschon letzteres für eine dritte Periode Wittgensteins sprechen könnte, werde ich zum Schluss doch einige früheren Aussagen von ihm anführen, die auf die Kontinuität seiner Konzeption hinweisen.

\section{Die Aussagen des Wissens und der Grammatik}

In seinen letzten Ausführungen stellt Wittgenstein zwei Arten von Aussagen einander gegenüber, die sich zwar beide scheinbar auf Fakten beziehen, in der Tat aber gilt die eine nicht als Erfahrungssatz, sondern als grammatischer Satz. Für einen solchen erscheint für Wittgenstein als Paradebeispiel die Mooresche Behauptung „Dies ist eine Hand“. Sie zählt nämlich zu unseren „fundamentalen“ Urteilen, deren „Wahrheit“ „zu unserm Bezugssystem“ gehört (MS 174: 17v). Infolgedessen können sie sich aber im eigentlichen Sinne nicht als falsch erweisen, bzw. wir können uns in ihnen nicht irren. Die Wörter „Irrtum“, „wahr“ und „falsch“ haben in Bezug auf sie keinen Sinn. Trotz ihrer Erfahrungssatz-Form beschreiben diese Sätze ein „Weltbild“, gehören „zu einer Art Mythologie“, und „ihre Rolle“ ist „der von Spielregeln“ ähnlich (siehe: MS 174: 21v) - ihre Rolle im Sprachspiel ist also den grammatischen Sätzen analog. Infolgedessen sagen sie nichts über die Wirklichkeit aus. Das Gesagte hat auch zur Folge, dass sich ein diesbezüglicher Fehler nicht in das Sprachspiel einordnen lässt: Schon ein einziger Fehlurteil gilt als Regelverstoß, mit dem man sich außerhalb des Sprachspiels ,hinausstößt“. Stellen wir also fest, dass jemand von uns in 
einem von unseren fundamentalen Urteilen abweicht, so wird er für uns als abnormal, als geistesgestört erscheinen, den wir nicht einmal richtig verstehen können. Das heißt auch, dass wir in diesen Fällen nicht geneigt sind, auch nur in Erwägung zu ziehen, dass er vielleicht Recht hat: Dass wir uns in einigen Urteilen nicht irren können, bedeutet nämlich nicht, dass kein Fehlurteil möglich ist, sondern es charakterisiert unsere Einstellung zu diesen Sätzen. Es heißt, dass sie für uns unumstößlich sind, egal, was auch passiert: Nichts auf der Welt kann uns vom Gegenteil überzeugen, und so auch keine Tatsachen, Gegenbeweise können diese Urteile falsifizieren.

Den fundamentalen, mythologischen Urteilen stehen die empirischen Sätze, die Aussagen des Wissens gegenüber: Diese können schon sowohl wahr als auch falsch sein; was aber als „Irrtum“, „wahr“ und „falsch“, und was als „Wirklichkeit“ und „Übereinstimmung mit der Wirklichkeit" gilt, wird vom Sprachspiel definiert. Somit haben wir aber selbst über die Erfahrungssätze keinen Zugang oder wenigstens nicht direkt zur Wirklichkeit. In ihnen können wir uns aber jedenfalls irren, und sogar mehrmals, ohne Gefahr zu laufen, dass man uns als Narren betrachtet. Ein Irrtum ist hier nämlich keine Unregelmäßigkeit, die nur ausnahmsweise vorkommt, und er bereitet auch keine Verständnisschwierigkeiten: Für ihn ist im Sprachspiel ein Platz vorgesehen - er lässt sich ohne weiteres in das Sprachspiel einordnen. Irrtümer sind daher diskutabel und lassen sich auch korrigieren - u.a. dadurch, dass wir unsere Aussagen mit der - vom Sprachspiel definierten - Wirklichkeit vergleichen. Wahrheiten sind auf der anderen Seite nicht unumstößlich: Es ist normal, dass man bereit ist, sich seine Aussagen nochmals zu überlegen und als Irrtümer aufzugeben, und dazu kann dadurch der Anstoß gegeben werden, dass jemand anderer Meinung ist. Irrt sich jemand aber all zu oft, dann ist das trotzdem kein Irrtum mehr, sondern zeugt vom Mangel am Verständnis des Sprachspiels; es zeigt, dass der Betreffende nicht imstande ist, sich unser Sprachspiel anzueignen. So einen Menschen werden wir nicht mehr verstehen, sondern er wird in unseren Augen zum Narren und Ketzer - ebenso, wie jemand, der mit uns ein fundamentales Urteil nicht teilt.

Bis zu diesem Punkt ist es nicht zu sehen, wo die Dinge „selbst“ in dem Sprachspiel eine konstitutive Rolle spielen, bzw. dass ihr So- oder Anders-Sein auf unsere Begriffe und Sprachspiele einwirken könnten. Es gibt aber trotzdem einen Platz für sie, der über die Irrtümer auf der Seite 
der Erfahrungssätze und über die Fehlurteile auf der Seite der fundamentalen Wahrheiten eröffnet wird.

Was die letzteren betrifft, kann es trotz dem oben Gesagten vorkommen, dass jemand seinen unerschütterlichen Glauben an der einen oder anderen fundamentalen Wahrheit aufgibt. Das wird aber sein ganzes System, sein ganzes Weltbild erschüttern, auch alle seine anderen Urteile mitreißen (MS 175: 75v) und ,alles [...] in ein Chaos [...] stürzen“ (MS 176: 75r). Dann wird er „,vor dem Nichts“ (MS 175: 59r), d.h. sozusagen ohne Sprachspiel, hilflos da stehen. Das wäre also ein Augenblick, in dem ihm die Tatsachen nicht vom Sprachspiel konstituiert erscheinen und zu Wort kommen könnten. Passiert dies nur einem einzigen Menschen, so gefährdet es das Sprachspiel selbst noch nicht. Wie aber, wenn viele Menschen sich gezwungen fühlten, ihre fundamentalen Urteile aufzugeben? Das würde das Sprachspiel schon aufheben und möglicherweise - wenigstens für eine Übergangsperiode, bis sich neue Gewohnheiten und Sprachspiele etablieren - den Dingen selbst wieder Spielraum lassen, und zwar diesmal nicht nur aus der Perspektive des Einzelnen, sondern auch aus jener der Sprachgemeinschaft.

Was die Irrtümer auf der anderen Seite betrifft, räumt Wittgenstein ohne weiteres ein, dass sich die Sprachspiele mit der Zeit bemerkt oder unbemerkt verändern, und u.a. eben dadurch, dass sich allmählich immer mehr Aussagen des Wissens ändern (z.B. MS 137: 74b, TS 232: 771, TS 233a: 72). Letzteres mag aber noch innerhalb der Sprachspiele bleiben, weil es nicht zwingend ist, anzunehmen, dass die Veränderung der einzelnen Aussagen dadurch bedingt ist, dass man die Aussagen an die Tatsachen der Welt angepasst hat: Wir haben ja gesagt, dass selbst die „Übereinstimmung mit der Wirklichkeit" vom Sprachspiel definiert ist. Wie aber, wenn nicht nur einige, sondern viele Leute, in vielerlei Hinsichten, und zwar im Laufe einer kurzen Periode ihre Meinungen änderten? Das kann schon das Sprachspiel selbst wieder - ähnlich wie im obigen Fall - erschüttern und aufheben, und infolgedessen uns auch die Dinge selbst erblicken lassen. 


\section{Können Tatsachen eine Veränderung unserer Begriffe bewirken?}

Wie gesagt, die fundamentalen Urteile sind dadurch charakterisiert, dass man sie unter keinen Umständen ändern muss. So werden sie auch nicht von einzelnen Beobachtungen in Zweifel gezogen. Wittgenstein spielt allerdings die Möglichkeit durch, wie es wäre, wenn unerwartet, ohne jeglichen Grund, etwas äußerst Außerordentliches passieren würde oder wenn zuviel Unerwartetes, was nicht mehr unseren, vom Sprachspiel bedingten Erwartungen entspricht, geschieht:

Wie, wenn etwas wirklich unerhörtes geschähe? Wenn ich etwa sähe, wie Häuser sich nach und nach ohne offenbare Ursache in Dampf verwandelten; wenn das Vieh auf der Wiese auf den Köpfen stünde, lachte und verständliche Worte redete; wenn Bäume sich nach und nach in Menschen, und Menschen in Bäume verwandelten? Hatte ich nun recht, als ich vor allen diesen Geschehnissen sagte "Ich weiß, daß das ein Haus ist" etc., oder einfach "Das ist ein Haus" etc.?

Diese Aussage erschien mir als fundamental; wenn das falsch ist, was ist noch 'wahr' und 'falsch'?! (MS 176: 43v-44r)

Wie der letzte Satz nahelegt, ist die erste Möglichkeit, die eintreten kann, der oben erwähnten ähnlich, nämlich dass dadurch alle Urteile mitgerissen werden und alles in ein Chaos gestürzt wird: Es ist offensichtlich durchaus vorstellbar, dass ich ,durch gewisse Ereignisse in eine Lage versetzt [würde], in der ich das alte Spiel nicht mehr fortsetzen könnte. In der ich aus der Sicherheit des Spiels herausgerissen würde“ (MS 176: 75v-76r).

Tatsachen können also unmöglich machen, dass ein Sprachspiel weiter gespielt wird. Das passiert aber nicht notwendigerweise: Alles kann wenigstens dem Anschein nach ebensogut auch unverändert bleiben. Auf die Frage nämlich,

Aber wäre es denn undenkbar, daß ich im Sattel bliebe, auch wenn die Tatsachen noch so sehr bockten? (MS 176: 75v)

gibt Wittgenstein eine bejahende Antwort:

Wenn auch plötzlich eine Unregelmäßigkeit im Naturgeschehn einträte, so müßte das mich nicht aus dem Sattel heben. Ich könnte, nach wie vor, Schlüsse machen - aber ob man das nun "Induktion" nennen \{wird / würde\}, ist eine andre Frage. (MS 176: 76r-76v) 
Der letzte Satz des Zitats weist allerdings darauf hin, dass das scheinbar unveränderte Sprachspiel unter den veränderten Umständen in der Tat schon ein anderes geworden ist.

Das Fazit dieses Gedankenexperiments ist also, dass „\{ein Sprachspiel / die Möglichkeit des Sprachspiels\} “, ob in dem ersten oder in dem zweiten vorhin genannten Sinne, aber , \{nur durch gewisse Tatsachen möglich ist / durch gewisse Tatsachen bedingt ist \}" (MS 176: 76r).

\section{Farben und Farbwörter}

Wittgenstein stellt im Zusammenhang mit den Farben und Farbwörtern in vielen Bemerkungen weitere Gedankenexperimente an, mittels welchen er auf die Frage eine Antwort sucht, ob es von den Tatsachen abhängt, welche Begriffe wir zu bilden und welche Sprachpiele wir zu spielen imstande sind.

Wittgensteins Gedankenexperimente spielen sich in fiktiven Ländern ab. In dem ersten Experiment lässt er uns ein Land vorstellen, „,wo die Farben der Dinge [...] unaufhörlich wechselten“ (MS 136: 51b; TS 232: 654), oder aber „wo alles nur eine Farbe hätte“ (MS 136: 52a). Dabei stellt er fest, dass man den Leuten dort den Gebrauch der Farbwörter nicht beibringen könnte, außerdem hätten dort unsere Farbwörter auch keine Anwendung, ließen sich dort unsere Sprachspiele nicht spielen. In einem anderen fiktiven Land ist die Farbenwelt hingegen ganz der unseren ähnlich, dafür sind aber die Leute dort entweder farbenblind oder sogar blind. Unsere Farbbegriffe und gewohnten Sprachspiele könnten also zwar in diesem Lande nur wegen der Farbenwelt ihre Anwendung haben, wir könnten aber trotzdem nicht den Einwohnern den Gebrauch unserer Farbwörter oder zumindest den Gebrauch einiger von ihnen beibringen; in diesem Lande gäbe es unser Farbsystem, einige unserer Sprachspiele nicht. Auf der anderen Seite würden wir selbst zumindest einige Schwierigkeiten damit haben, uns die Sprache dieser Leute anzueignen (MS 173: 30v). Sowohl für das Land ohne Farben bzw. mit den sich fortwährend ändernden Farben, als auch für jenes der Blinden bzw. Farbenblinden trifft die Behauptung zu, dass ,hier zwischen logischer und physischer Möglichkeit der Unterschied nicht gesehen“" wird (MS 136: 52a; TS 232: 654). 
Die physikalische Beschaffenheit der Welt hat also beide Male Folgen bezüglich dessen, ob ein Sprachspiel überhaupt gespielt wird: Unsere Begriffe, unsere Sprache sind gegenüber diesen ,harten“ Fakten sozusagen machtlos.

Stellen wir uns nun das Gegenteil der obigen beiden Beispiele vor: ein Land, in dem sich unsere Farbwörter reibungslos anwenden lassen, und in dem auch das Sehvermögen der Leute dem unseren entspricht - wo sich aber die Farbbebriffe von den unseren unterscheiden. Die Leute dort wären also - im Gegensatz zu den ,natürlichen“ Farbenblinden - sozusagen aus „begrifflichen“ Gründen farbenblind. Die Auslegung dieser Situation ist bei Wittgenstein nicht eindeutig: Auf die Frage, ob wir die Farbbegriffe dieses Landes als Farbbegriffe erkennen würden, neigt er eher - obschon manchmal mit Einschränkungen - zu einer verneinenden Antwort (MS 173: 11r-11v, 29v-30r; MS 176: 5r). Auf die Frage, ob wir diesen Leuten unsere Farbbegriffe beibringen bzw. ob wir uns ihr Farbsystem aneignen könnten, schwankt seine Antwort hingegen zwischen Ja und Nein (MS 133: 17r-18v; MS 137: 101a; TS 229: 327-328, 342; TS 233a: 70). Die Macht der Sprache scheint also zumindest in einigen Fällen nicht eindeutig bestimmend zu sein.

Das Fazit der drei Gedankenexperimente ist nun das Folgende: 1.) Daraus, dass sowohl die „natürlichen“ als auch die „begrifflichen“ Umstände dieselben Konsequenzen haben können, nämlich, dass einige Sprachspiele nicht gespielt werden bzw. nicht gespielt werden können, ergibt sich der Schluss, dass die Dinge der Welt, zumindest in manchen Fällen, nicht weniger bestimmend ist, als die Sprache. 2.) Der dritte Fall - in dem es nicht auszuschließen war, dass man die Grenzen der eigenen Sprachspiele überschreiten kann - legt sogar nahe, dass die Macht der Fakten in einigen Fällen sogar überwältigender ist als jene der Sprache.

\section{Konklusion}

Mit der Formulierung der Schlussfolgerung, dass die Sprachspiele durch gewisse Tatsachen bedingt sein müssten, ist Wittgenstein aber offensichtlich unzufrieden und versucht immer wieder, diese Idee neu zu formulieren. Der Grund seiner Unzufriedenheit mag darin liegen, dass die Behauptung eine wissenschaftliche Hypothese, bzw. das Wort „bedingt“ einen kausalen 
Zusammenhang nahelegen, und Hypothesen und kausale Zusammenhänge werden von Wittgenstein nicht als geeignete Gegenstände einer philosophischen Untersuchung akzeptiert. Eine Formulierung dieser Art verwischt den Unterschied zwischen der physischen und logischen Möglichkeit bzw. Unmöglichkeit. Uns interessiert aber eben nicht,

unter welchen Umständen das Sprachspiel mit den Farbnamen physisch nicht möglich - also eigentlich, nicht wahrscheinlich ist.

Ohne Schachfiguren kann man nicht Schach spielen - das ist die Unmöglichkeit, die uns interessiert. (TS 232: 654-655, vgl. MS 136: 52a)

Als Ausweg aus diesem Dilemma bietet sich erstens an, dass man einfach feststellt: Es ist eine alltägliche Erfahrungstatsache, dass Sprachspiele nur unter bestimmten Umständen gespielt werden, bzw. dass die Veränderungen der Dinge öfters mit Veränderungen der Sprachspiele einhergehen oder von ihnen gefolgt werden (z.B. MS 137: 74a; TS 232: 771). Somit wird also bloß eine Tatsache unserer Sprachspiele festgelegt. Das heißt, dass wir mit so einer Behauptung nichts anderes tun, als dass wir unsere Sprachspiele beschreiben - und das lässt sich schon ohne weiteres in die Wittgensteinsche Konzeption einordnen. Eine andere Lösungsmöglichkeit bieten die folgenden Ausführungen an:

Wenn du dir gewisse Tatsachen anders denkst, sie anders beschreibst, als sie sind, dann kannst du die Anwendung gewisser Begriffe dir nicht mehr vorstellen, weil die Regeln ihrer Anwendung kein Analogon unter den neuen Umständen haben. - Was ich sage, kommt also darauf hinaus: Ein Gesetz wird für Menschen gegeben und ein Jurist mag wohl fähig sein, Konsequenzen für jeden Fall zu ziehen, der ihm gewöhnlich vorkommt, das Gesetz hat also offenbar seine Verwendung, einen Sinn. Trotzdem aber setzt seine Gültigkeit allerlei voraus; und wenn das Wesen, welches er zu richten hat, ganz vom gewöhnlichen Menschen abweicht, dann wird z.B. die Entscheidung, ob er eine Tat mit böser Absicht begangen hat, nicht etwa schwer, sondern (einfach) unmöglich werden. (TS 232: 705; vgl. MS 136: 121a)

Der erste Satz des Zitats führt den darauf folgenden Fall als Gedankenexperiment ein, das als solches nicht mehr auf kausale, i.e. externe, sondern auf nicht-kausale, interne Eigenschaften gerichtet ist. Diese Verfahrensweise lässt sich mit jener vergleichen, die Wittgenstein als „Naturtatsachen erdichten" bezeichnet, und das heißt, dass man, anstatt eine kausale, naturgeschichtliche Untersuchung durchzuführen, die Tatsachen anders denkt, 
sie sich anders vorstellt, als sie sind, um dadurch seine Augen auf gewisse nicht-kausale Zusammenhänge schärfer zu machen.

Wenn also Wittgenstein die Formulierung, die Möglichkeit des Sprachspiels sei durch gewisse Tatsachen bedingt, als unangebracht ablehnt, so tut er es nicht, weil er ihnen gar keine konstitutive Rolle zusprechen möchte. Die letztere Idee fügt sich sogar problemlos in die Sprachspiel-Konzeption ein. Wittgenstein hat den Sprachspiel-Begriff u.a. eingeführt, um hervorzuheben: Die Worte funktionieren nur, wenn sie in Handlungen, in einem im weitesten Sinne genommenen Kontext und in einer Umgebung eingebettet sind. Somit hat er in den Begriff des Sprachspiels nicht nur die Sprache selbst, sondern auch die Handlungen, Gesten, den Kontext und die Umgebung einbezogen. Unter den Begriff der Umgebung müssen freilich auch gewisse Tatbestände - ihr Vorhandensein oder Nichtvorhandensein, ihre Beschaffenheiten, so auch die Eigenschaften der physikalischen Gegenstände - fallen. Wenn aber die Dinge der Welt auf diese Weise im Sprachspiel inbegriffen sind, dann ist es auch kein Wunder, dass ihre Veränderungen mit einer Veränderung des Sprachspiels einhergehen können. Mehr noch: Gelten die Dinge selbst als Komponenten der Sprachspiele, dann sind auch keine außerordentlichen Umstände dazu nötig, dass sie im Sprachspiel „mitreden“ können (vgl. MS 137: 7b; TS 232: 714; TS 233b: 2), sondern sie kommen auch unter normalen Umständen zu Wort, nicht ungleich, wie die Komponenten, die mit der menschlichen Kultur und Sprache zu tun haben. Wittgensteins mit außerordentlichen Umständen operierende Gedankenexperimente dienen also nur dem Zweck, mit Hilfe des Ungewöhnlichen alltägliche Zusammenhänge zu Tage zu bringen.

Fassen wir nun mit Hilfe eines einfachen Beispiels das Fazit unserer Ausführungen zusammen. Nehmen wir das Tennisspiel: Entscheiden wir uns - obschon alle äußeren Umstände wie gewöhnlich sind -, Tennis nach anderen Regeln zu spielen als üblich und im Regelverzeichnis vorgeschrieben, so spielen wir kein Tennis mehr. Steht uns hingegen plötzlich kein Tennisball mehr zur Verfügung, sondern nur noch ein Fußball, so können wir das Spiel entweder nicht oder nur ganz anders als üblich spielen - und so spielen wir wieder kein Tennis mehr. Hätten wir nie im Leben Tennisbälle, sondern nur Fußbälle zur Verfügung gehabt, so hätten wir die Regeln des Spiels schon von Anfang an anders gebildet. Unsere Regeln richten sich nämlich u.a. nach den Möglichkeiten: Kein Aufschlag wird 
z.B. vorgeschrieben, der über die menschlichen Kräfte geht. (Oder wenn so einer trotzdem vorgeschrieben würde, dann würde so eine nicht durchführbare Anweisung im Sprachspiel eine andere Rolle spielen, als die Regeln, die sich auch befolgen lassen.) Diese Lehre wird auch im obigen Zitat vermittelt, indem es darauf hinweist: Die menschlichen Gesetze funktionieren nur unter Menschen und haben keine Verwendung unter Wesen, die sich von uns wesentlich unterscheiden.

Abschließend möchte ich diese Schlussfolgerungen noch mit zwei weiteren Textstellen belegen, wobei ich kurz auch die Frage eines „Third Wittgenstein" thematisieren werde.

\section{Third Wittgenstein}

Das vorhin Gesagte, nämlich, dass die aufgrund der Textstellen aus den Jahren 1946-51 dargestellte Wittgensteinsche Auffassung die sprachspielkonstituierende Rolle der Tatsachen betreffend ohne Schwierigkeiten der Sprachspielkonzeption eingliedern lässt, deutet schon darauf hin, dass sich die Konzeption Wittgensteins in der Periode nach den Philosophischen Untersuchungen zumindest in dieser Hinsicht nicht wesentlich von seiner früheren unterscheidet. Die Idee geht in der Tat sogar auf eine lange Zeit zurück, wie es dem folgenden Zitat entnehmen lässt:

So lernen ja die Kinder bei uns rechnen, denn man läßt sie drei Bohnen hinlegen und noch drei Bohnen und dann zählen, "was da liegt". Käme dabei einmal 5 einmal 7 heraus (weil wie wir jetzt sagen würden einmal von selbst eine dazu, einmal eine weg käme), so würden wir zunächst Bohnen als für den Rechenunterricht ungeeignet erklären. Geschähe das Gleiche aber mit Stäben, Fingern, Strichen und den meisten andern Dingen, so hätte das Rechnen damit ein Ende.

"Aber wäre dann nicht doch noch $2+2=4$ ?" - Dieses Sätzchen wäre damit unbrauchbar geworden. (MS 118: 32v)

Diese, zuerst Ende August 1937 notierte Bemerkung wurde von Wittgenstein im September nochmals in MS 117: 44, sodann 1938 in TS 221: 170 geringfügig weiterbearbeitet, bis sie schließlich noch in demselben Jahr in die Frühfassung der Philosophischen Untersuchungen Eingang gefunden hat (PU-KGE, 358). Irgendwann zwischen 1939 und 1943 hat Wittgenstein dann die Frühfassung neu bearbeitet, dabei TS 221 in Zettel zerschnitten 
und von diesen TS 222 zusammengestellt. TS 222 diente als Grundlage des zweiten Teils der bearbeiteten Frühfassung der Philosophischen Untersuchungen. Die obige Bemerkung kommt in den beiden vor (TS 222: 31; BGM, 51-52). ${ }^{1}$ Die Frühfassung hat Wittgenstein im Sommer 1938, die bearbeitete Frühfassung im September 1943 bei dem Cambridge University Press unter dem Titel Philosophische Bemerkungen eingereicht, es sich aber später beide Male anders überlegt und sein Publikationsvorhaben zurückgezogen. Die beiden Frühfassungen, die auch noch auf mehrere Vorstufen mit dem gleichen Inhalt zurückgehen, fallen dennoch schwer genug ins Gewicht, um auf ihrer Grundlage zweifellos behaupten zu können, dass Wittgensteins Auffassung schon zu dieser Zeit der späteren ähnlich gewesen ist: $\mathrm{Zu}$ dem obigen Fall mit den immer wieder verschwindenden Bohnen hätte er schon, ebensogut wie später die Schlussfolgerung hinzufügen können, dass hier kein Unterschied zwischen logischer und physischer Unmöglichkeit zu sehen ist.

Nach der bearbeiteten Frühfassung nahm Wittgensteins Arbeit an den Philosophischen Untersuchungen eine andere Richtung, wobei er die Bemerkungen zu Fragen der Mathematik, in denen die obigen Zeilen ihren Platz gehabt hatten, fallen ließ. Infolgedessen ist die Bemerkung in der Zwischenfassung und somit freilich auch in der Spätfassung der $P U$ nicht mehr zu lesen. Die Position aber, die in ihr zum Ausdruck gekommen ist, bleibt weiterhin aufrechterhalten, wie davon etwa $\$ 142$ der PU zeugt:

Und verhielten sich die Dinge ganz anders, als sie sich tatsächlich verhalten [...] so verlören unsere normalen Sprachspiele ihren Witz. - Die Prozedur, ein Stück Käse auf die Waage zu legen und nach dem Ausschlag der Waage den Preis zu bestimmen, verlöre ihren Witz, wenn es häufiger vorkäme, daß solche Stücke ohne offenbare Ursache plötzlich anwüchsen, oder einschrumpften. (PU, §142)

Zwischen den Konzeptionen vor und nach den Philosophischen Untersuchungen ist also, was zumindest die konstitutive Rolle der Tatsachen betrifft, kein wesentlicher Unterschied zu sehen. Wollen wir mit dem Termin „Third Wittgenstein“ nicht nur auf Wittgensteins verstärktes oder schwächeres Interesse für gewisse Fragen oder bloß auf eine weitere Nuancie-

${ }^{1}$ Der erste Teil der bearbeiteten Frühfassung ist in PU-KGE, der zweite als Teil I der Bemerkungen über die Grundlagen der Mathematik erschienen. 
rung und Vertiefung von früheren Konzepten, sondern auch auf konzeptionelle Unterschiede hinweisen, so gibt es wenigstens im Hinblick auf diese Frage keinen „Third“ Wittgenstein. Damit will ich allerdings nicht behaupten, dass es überhaupt keinen gibt: Der Umstand, dass sich der früher als Teil II der PU, inzwischen als Philosophie der Psychologie - Ein Fragment herausgegebene Text konzeptionell schwerlich an die Stelle der letzten ca. 170 Paragraphen der PU einarbeiten lässt (Neumer 2000, 243f., fn15), wie es noch die Herausgeber der Erstauflage gedacht haben, bzw. dass „das Buch [...] im Falle einer solchen Überarbeitung ein völlig anderes geworden" wäre (Schulte 2001, 30), legt schon nahe, dass man das Konzept nicht so leicht vom Tisch haben kann. Diese Fragen gehören aber bereits zu einer anderen Untersuchung.

\section{Literatur}

Neumer, Katalin 2000: Die Relativität der Grenzen: Studien zur Philosophie Wittgensteins. Amsterdam/Atlanta, GA.

Schulte, Joachim 2001: „Einleitung“, in: PU-KGE, 12-50.

Wittgenstein, Ludwig 1984: Bemerkungen über die Grundlagen der Mathematik. In: Werkausgabe in 8 Bänden, Bd. 6. Frankfurt: Suhrkamp. (BGM)

Wittgenstein, Ludwig 2000: Wittgenstein's Nachlass: The Bergen Electronic Edition. Oxford: Oxford University Press. (BEE) ${ }^{2}$

Wittgenstein, Ludwig 2001: Philosophische Untersuchungen: Kritisch-genetische Edition, hrsg. v. Joachim Schulte in Zusammenarbeit mit Heikki Nyman, Eike von Savigny und Georg Henrik von Wright. Frankfurt am Main: Suhrkamp. (PU-KGE)

Wittgenstein, Ludwig 2003: Philosophische Untersuchungen, auf der Grundlage der Kritisch-genetischen Edition neu hrsg. v. Joachim Schulte. Frankfurt: Suhrkamp. (PU)

${ }^{2}$ Die Manu- und Typoskripte Wittgensteins werden auf der Grundlage der BEE zitiert. Ich zitiere zwar die „normalisierte” Version der BEE, ergänze sie aber aufgrund der Faksimileaufnahmen der BEE mit allen Textvarianten, die Wittgenstein nicht gestrichen, sondern stehen gelassen hat. Ich habe in den Zitaten die Transkription der BEE überprüft und die Transkriptionsfehler stillschweigend korrigiert, darüber hinaus Wittgensteins Leerzeilen wiederhergestellt.

Textvarianten werden in geschwungenen Klammern $\{\ldots / \ldots\}$ und unterstrichene in kursiver Schrift wiedergegeben. In den angeführten Zitaten verwendet Wittgenstein keine von seinen anderen üblichen Hervorhebungsarten. 